\title{
Physiochemical Properties of Binary Pluronic Systems for Reversal of Multi-drug Resistant (MDR) Cancers
}

\author{
Jungmin Yun ${ }^{1}$, Gayoung Park ${ }^{1}$, HaHyung Kim¹, Jaehwi Lee ${ }^{1}$, EunSeong Lee ${ }^{2}$, Yu Seok Youn ${ }^{3}$, Beom-Jin Lee ${ }^{4}$, \\ Young Taik $\mathrm{Oh}^{5 \dagger}$ and Kyung Taek $\mathrm{Oh}^{1 \dagger}$ \\ ${ }^{1}$ College of Pharmacy, Chung-Ang University, 221 Heukseok dong, Dongjak-gu, Seoul 155-756, Republic of Korea \\ ${ }^{2}$ Division of Biotechnology, The Catholic University of Korea, 43-1 Yeokgok 2-dong, Wonmi-gu, Bucheon-si, \\ Gyeonggi-do 420-743, Republic of Korea \\ ${ }^{3}$ College of Pharmacy, Pusan National University, Jangjun-dong, Geumjeong-gu, Busan 609-735, Korea \\ ${ }^{4}$ Bioavailability Control Laboratory, College of Pharmacy, Kangwon National University, Chuncheon, Korea. \\ ${ }^{5}$ Department of Diagnostic Radiology, Research Institute of Radiological Science, College of Medicine, \\ Yonsei University Seodaemun-kuShinchon-dong 134, Seoul, 120-752, Republic of Korea
}

(Received July 21, $2010 \cdot$ Revised August 12, 2010 • Accepted August 13, 2010)

\begin{abstract}
Pluronic as pharmaceutical excipients are listed in the US and British Pharmacopoeia. In particular, Pluronics exist as different compositions and display abundant phases as self-assembling into polymeric micelles with various morphologies depending on the aqueous solvent quality, the composition of structure, and hydrophilic-lipophilic balance (HLB). Pluronics were also known as a P-gp modulator, which was exploited as a reversal molecule of multi-drug resistant (MDR) cancers. We selected a lamella forming Pluronic L92 which has high hydrophobicity and relatively long PEO block among L series of Pluronics. The dispersion of L92 showed great size particles and low stability. To increase the stability and to decrease the particle size, secondary Pluronics (F68, F88, F98, F127, P85, P105, and P123) with relatively long PEO chain were added into $0.1 \mathrm{wt} \%$ Pluronic L92 dispersion. The stability of binary systems was increased due to incorporated long PEO chain. Their particle sizes slightly decreased to over $200 \sim 400 \mathrm{~nm}$ and their solubilization capacity of binary systems didn't change except Pluronic L92/P123 mixtures. The L92/P123 systems showed ca. $100 \mathrm{~nm}$ sizes and lowest turbidity among the all systems. The solubilization capacity of $0.1 \mathrm{wt} \% \mathrm{~L} 92 / 0.1 \mathrm{wt} \% \mathrm{P} 123$ was slightly increased compared to $0.1 \mathrm{wt} \%$ L92 mono system and other binary systems. These nano-sized binary systems may have potential as alternative drug delivery systems with simple preparation method and overcome the drawbacks of mono systems such as low stability and loading capacity.
\end{abstract}

Key words - Pluronic, binary system, nanosized, MDR, drug delivery system, solubilization

Recently, polymer-based drug delivery and drug targeting systems have a tremendous influence on development of new drug therapies (Langer 1998). Soluble amphiphilic block copolymers can self-assemble into supramolecular structures, which were studied for nano-carriers of anticancer drugs, proteins, and genes (Kwon and Kataoka 1995; Allen et al., 1999; Kataoka and Kabanov 1999), as well as imaging agents (Torchilin 1999). Pluronic, consisting of poly(ethylene oxide) (PEO) and poly(propylene oxide) (PPO) blocks with a PEOPPO-PEO arrangement (often as $\mathrm{EO}_{\mathrm{x}}-\mathrm{PO}_{\mathrm{y}}-\mathrm{EO}_{\mathrm{x}}$ ) have been studied for pharmaceutical applications such as solubilization excipients, drug carriers, immunoadjuvants, cryopreservation agents, and gene carriers (Allison and Byars 1986, 1990; Alakhov et al., 2001; Batrakova et al., 2001; Kabanov and Alakhov

†Corresponding Author:

Tel : +82-2-824-5617, E-mail : kyungoh@cau.ac.kr

DOI : 10.4333/KPS.2010.40.4.255
2002; Oh et al., 2004; Oh et al., 2008). This arrangement results in an amphiphilic copolymer in which the number of hydrophilic $\mathrm{EO}_{\mathrm{x}}$ and hydrophobic $\mathrm{PO}_{\mathrm{y}}$ units can be altered to vary the size, morphology, hydrophilicity, and lipophilicity.

A defining property of Pluronic is micellization defined as a process to form aggregation from individual block copolymer molecules, unimers at concentration of Pluronic above the critical micelle concentration (CMC) and critical micelle temperature (CMT). Pluronic micelles (above 30\% PEO content) are commonly pictured as spheres composed of a PPO core and a PEO corona. However, cylindrical and lamellar structures are also included in micelle morphologies (Nagarajan, 1999). This morphology is affected by several factors such as Pluronic composition, concentration, temperature, and quality of the solvent (Allen et al., 1999; Kabanov and Alakhov 2002).

These copolymers such as Pluronic L92 (Pluronic L92 is designated as L92 throughout), P105, and F127 are commercially available from BASF and customized for applica- 
tions and customer needs. In the trade names, the letters stand for solid (F), paste (P), or liquid (L). The last digit of the number approximates the weight content of PEO block in tens of weight percent. The remaining first one or two digits encode the molecular mass of the central PPO block which can be obtained by multiplying the corresponding number by 300 . This nomenclature is widely used and helpful for predicting their behavior in solution.

Pluronics has used to interrupt the P-gp mediated drug efflux pump (Batrakova et al., 1999b; Kabanov et al., 2002). Pluronic formulations with anticancer drugs, below their CMC, have been claimed to be effective in treating MDR tumors by their crossing the plasma membrane and suppressing ATP production. The P-gp modulation and cytotoxicity for MDR cells increased with increase in the copolymer hydrophobicity (Batrakova et al., 1999b). This effect has also been linked with gene modulation by Pluronic block copolymers (Batrakova et al., 1999b; Kabanov et al., 2002). In addition, binary systems with two Pluronics have been researched to compensate for drawbacks of a mono system and control the physicochemical properties for smart drug delivery systems (Alakhov et al., 1999; Lee et al., 2003; Lee et al., 2005; Lee et al., 2010; Oh et al., 2004; Oh et al., 2008; Wang et al., 2007). For example, a mixture of hydrophobic L61 and hydrophilic F127 has practical application in pharmaceutics for a doxorubicin delivery system (Alakhov et al., 1999), and selection of the mixture components produced physicochemical properties such as a high colloidal stability, formation of small particle size, and good solubilization capacity. In particular, L121/F127 and L121/P123 systems produced nanosized stable systems with several-fold higher loading capacity in hydrophobic agents compared to F127 and P123 micelles (Oh et al., 2004).
In this paper, we have tested blend systems with L92 and several secondary Pluronics (Table I) (BASF; Kozlov et al., 2000). L92 has high hydrophobicity and is one of the Pluronic with relatively long PEO block among $\mathrm{L}$ series of Pluronics. L92 showed the inhibition of the P-gp efflux systems and high solubilization capacity. However, the hydrophobic Pluronics have a low aqueous stability. We tried to overcome the drawbacks of L92 such as low stability and relatively huge aggregations by addition of intermediate and hydrophilic Pluronics which have relatively long PEO chains compared to L92. We evaluated size, stability, turbidity and solubilization capacity of the blends using a model hydrophobic compound, Sudan III.

\section{Experimental}

\section{Materials}

Pluronics are commercially available from BASF Corporation (Parsipanny, NJ, USA) and were used without additional purification. The molecular characteristics of the block copolymers are presented in Table I. Sudan III (Samchun Co., Seoul, South Korea) was used as a hydrophobic model drug for solubility studies. All other chemicals were of analytical grade.

\section{Preparation of Binary Mixed Systems}

The stock solutions (2 10 wt \%) of Pluronics were prepared in distilled water and stored at $4^{\circ} \mathrm{C} .0 .01 \sim 0.05 \mathrm{wt} \%$ L92 dispersion were prepared by diluting the cold stock solution (2 $\mathrm{wt} \%$ ) with distilled water and stored in the refrigerator overnight. In case of the binary systems, the concentration of hydrophobic L92 was kept constant at $0.1 \mathrm{wt} \%$ while secondary Pluronics (F68, F88, F98, F127, P85, P105, and P123) was varied from 0.01 to $0.1 \mathrm{wt} \%$. Pluronic binary systems

Table I. Physicochemical Characteristics of Pluronics Used in this Study

\begin{tabular}{ccccccc}
\hline \hline Copolymer & MW & $\begin{array}{c}\text { Average no. } \\
\text { PEO units }(\mathrm{x})^{\mathrm{b}}\end{array}$ & $\begin{array}{c}\text { Average no. } \\
\text { PEO units }(\mathrm{y})^{\mathrm{b}}\end{array}$ & HLB $^{\mathrm{c}}$ & $\begin{array}{c}\text { Cloud point in 1\% w/v }_{\text {aqueous solution }\left({ }^{\circ} \mathrm{C}\right)^{\mathrm{c}}} \\
\text { (qMC(M) }\end{array}$ \\
\hline F68 & 8400 & 152.73 & 28.97 & 29 & $>100$ & $4.8 \times 10^{-4}$ \\
P85 & 4600 & 52.27 & 39.66 & 16 & 85 & $6.5 \times 10^{-5}$ \\
F88 & 11400 & 207.27 & 39.31 & 28 & $>100$ & $2.5 \times 10^{-4}$ \\
L92 & 3650 & 16.59 & 50.34 & 6 & 26 & $8.8 \times 10^{-5}$ \\
F98 & 13000 & 236.36 & 44.83 & 28 & $>100$ & $7.7 \times 10^{-5}$ \\
P105 & 6500 & 73.86 & 56.03 & 15 & 91 & $6.2 \times 10^{-6}$ \\
P123 & 5750 & 39.20 & 69.40 & 8 & 90 & $4.4 \times 10^{-6}$ \\
F127 & 12600 & 200.45 & 65.17 & 22 & $>100$ & $2.8 \times 10^{-6}$ \\
\hline
\end{tabular}

${ }^{a}$ Average molecular weights provided by the manufacturer (BASF Co, Parsippany, NJ) (BASF).

${ }^{\mathrm{b}}$ The average number of PEO and PPO units were calculated using the average molecular weights.

${ }^{\mathrm{c}} \mathrm{Hydrophilic-lipophilic}$ balance (HLB) values of the copolymers and the cloud points were determined by the manufacturer.

${ }^{\mathrm{d}}$ Critical micelle concentration (CMC) values were determined previously using pyrene probe (Kozlov et al., 2000). 
were prepared from stock solutions in the proportions indicated, and left at $4^{\circ} \mathrm{C}$ overnight to produce unimers. The solutions were transferred to room temperature $\left(23^{\circ} \mathrm{C}\right)$ and incubated for more than 1 hour to form the aggregates.

\section{Size Measurements Using Dynamic Light Scattering (DLS)}

The effective hydrodynamic diameter $\left(\mathrm{D}_{\text {eff. }}\right)$ of the particles was measured by photon correlation spectroscopy using a "Zetasizer Nano-ZS" (Malvern Instruments, UK) equipped with the Multi Angle Sizing Option (BI-MAS). The sizing measurements were performed in a thermostatic cell at a scattering angle of $90^{\circ}$. Software provided by the manufacturer

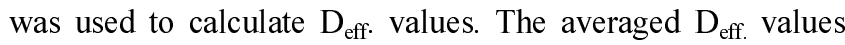
were calculated from three measurements performed on each sample $(n=3)$.

\section{Turbidity Measurements}

The turbidity experiments were performed by measuring the transmittance of the mixed samples using a Genesys 10UV (Thermo Sci., USA) at $\lambda=520 \mathrm{~nm}$. Before the test, all micelle solutions $(n=3)$ were stabilized at room temperature for $1 \mathrm{~h}$.

\section{Solubilization of Water-insoluble Dye}

Ten microliters of Sudan III stock solution $(10 \mathrm{mg} / \mathrm{mL})$ in $\mathrm{CHCl}_{3}$ was added to empty vials and the solvent was evaporated. Two milliliter aqueous solutions of binary mixture dispersions prepared were individually added to the vials and then allowed to equilibrate in the shaker $(100 \mathrm{rpm})$ at room tem- perature for 1 day. Absorption spectra of Sudan III in aqueous solutions at $25^{\circ} \mathrm{C}$ were recorded on a Genesys 10UV (Thermo Sci., USA). The data are reported as absorbance at $362 \mathrm{~nm}$ corresponding to the maximum in the Sudan III spectra.

\section{Results and Discussion}

\section{Formation of Binary System Using Pluronics}

Amphiphilic block copolymers have various phases depending on their nature, structure, and composition. Pluronic, symmetric triblock copolymers, displayed spherical, cylindrical, and lamellar aggregates according to relative length of $\mathrm{PEO}$ and PPO. $\mathrm{L} 92\left(\mathrm{EO}_{8}-\mathrm{PO}_{50}-\mathrm{EO}_{8}\right)$ with approximately $20 \% \mathrm{PEO}$ block and $80 \%$ PPO block have ca. $0.03 \% \mathrm{w} / \mathrm{v} \mathrm{CMC}$ at $37^{\circ} \mathrm{C}$ (Batrakova et al., 1999a). L92 forms a supra-macromolecular structure with a lamellar morphology because $20 \%$ PEO of the whole MW cannot provide sufficient steric hindrance of spherical formation. The lamella aggregation has high solubilization capacity but low aqueous stability (Nagarajan 1999). The size of L92 particle was average ca. $600 \mathrm{~nm}$ at $0.05 \%$ wt and very unstable. The data of DLS measurement showed size fluctuation and multimodal size distribution. As increase of L92 concentration, the aqueous dispersion turned cloud and precipitation in the dispersion was observed even though the size of particle appeared somewhat decreased and size distribution showed bi-modal distribution (Figure 1 and 2, (a)). Although the particle size in L92 dispersion prepared in this study appeared decreased and less fluctuated, the results that may be due to measurement of dispersion phase after precipitation. It (a)

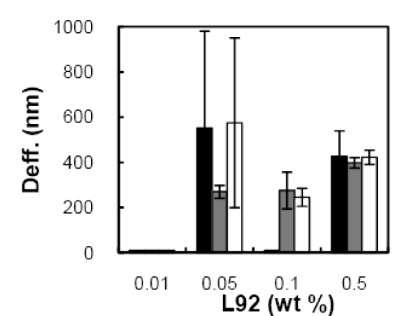

(e)

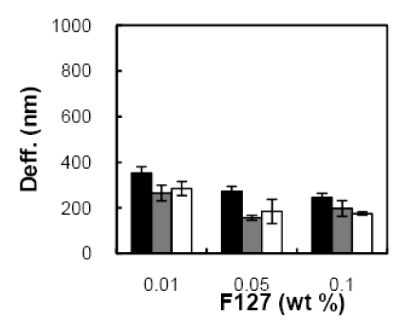

(b)

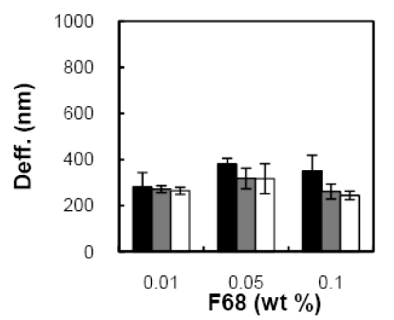

(f)

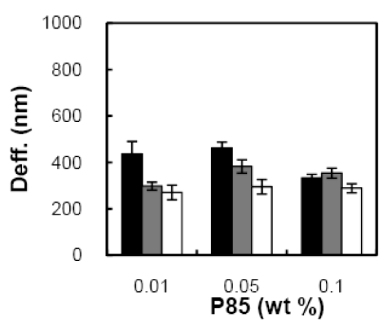

(c)

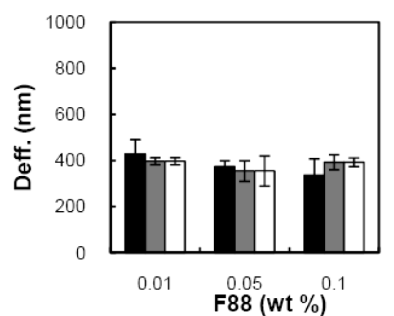

(g)

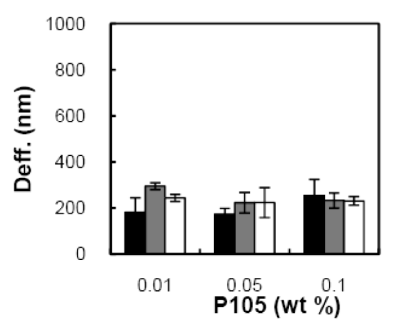

(d)

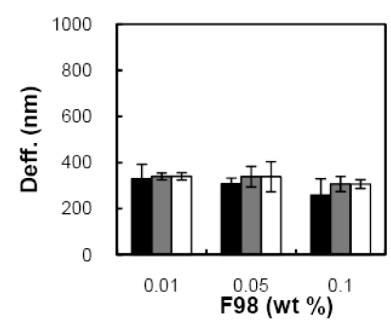

(h)

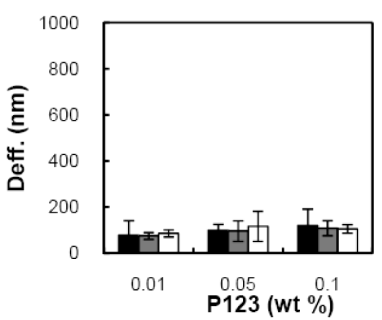

Figure 1. The particle size of different Pluronic dispersions as a function of the concentration at 1 hour (black bar), 12 hours (grey bar), and 24 hours (white bar): (a) mono system of L92 (b) (h) binary systems of 0.1 wt\% L92 admixed with (b) F68 (c) F88 (d) F98 (e) F127, (f) P85 (g) P105 and (h) P123. Each point represents average \pm SD (n=3). 
(a)

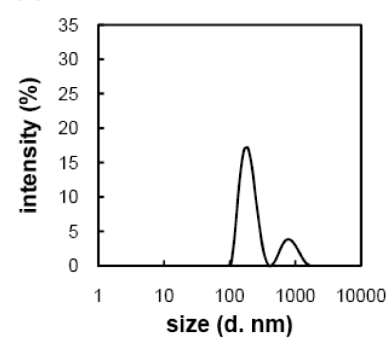

(e)

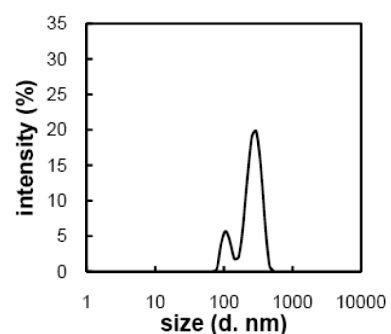

(b)

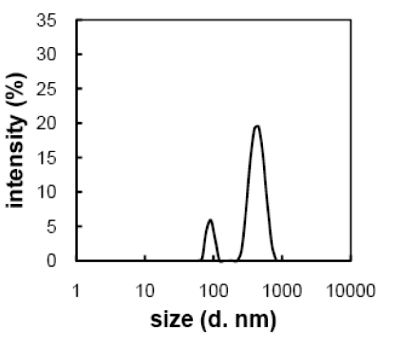

(f)

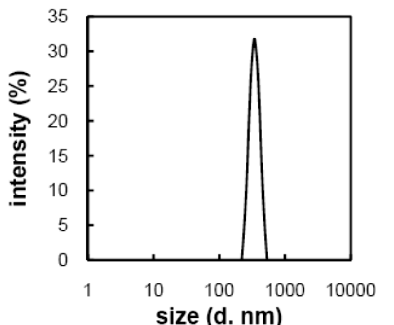

(c)

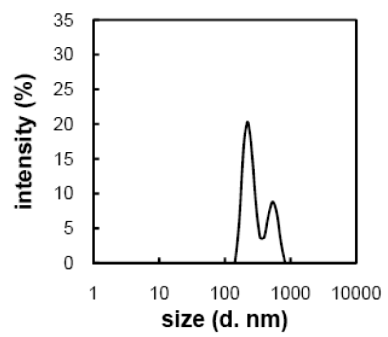

(g)

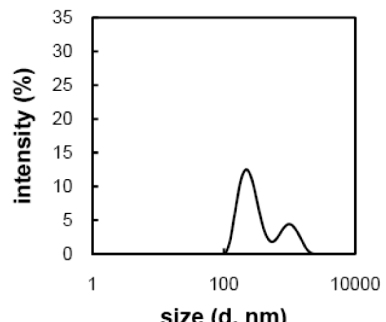

(d)

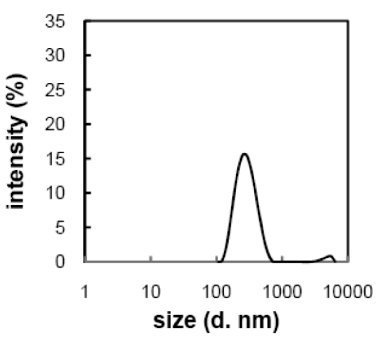

(h)

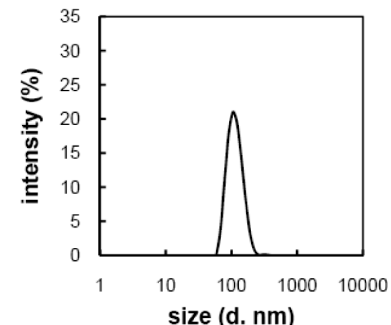

Figure 2. The particle size distribution of (a) 0.1 wt\% L92 and mixtures of 0.1 wt \% L92 and 0.1\% of (b) F68 (c) F88 (d) F98 (e) F127 (f) P85 (g) P105 and (h) P123.

indicated that the kinetic stability in particle of L92 displayed low.

To form a stable dispersion, other Pluronic with relatively long PEO chain was admixed into L92. Figure 1 ((b) (h)) represents the particle size of binary mixtures as a function of concentration of Pluronics. The concentration of hydrophobic L92 was kept constant at $0.1 \mathrm{wt} \%$, which showed less stability with fluctuation and bimodal distribution while the concentration of other Pluronics was varied from 0.01 to $0.1 \mathrm{wt} \%$. In case of Pluronics with long PEO chain, F68, F88, F98 and F127, the addition of hydrophilic Pluronics led to formation of $300 \mathrm{~nm}$ to $400 \mathrm{~nm}$ aggregation and increase of stability without precipitation for 24 hours (Figure 1, (b) (e)). The size distribution of binary systems was still broad and showed a changed bi-modal distribution at mixture with F68, F88, and F127 (Figure 2). Interestingly, the binary system with $0.1 \%$ L92 and $0.1 \%$ F98, however, demonstrated the uni-modal distribution (Figure $2(\mathrm{~d})$ ). This suggested that Pluronics with similar MW of PPO block can be blended and form a cooperative binding and those with different PPO block cannot form non-cooperative aggregation (Chaibundit et al., 2007; Gaisford et al., 1997; Oh et al., 2004). In addition, the increased stability of binary systems may be due to addition of hydrophilic Pluronic which can bind into PPO of L92 and provide the steric hindrance by increasing the corona forming PEO (Oh et al., 2004).

Addition of P85, P105, and P123 with intermediate hydrophilic-lipophilic balance (HLB) to L92 similarly resulted in decrease of particle size and increase of aqueous stability (Fig- ure 1. (f) (h)). The size distribution of L92/P85 and L92/ P123 were uni-modal and narrow while L92/P105 showed bimodal size distribution despite of $200 \mathrm{~nm}$ particle size (Figure 2. (f) (h)). Notably, L92/P123 systems represented less than $100 \mathrm{~nm}$ aggregates in all ranges of concentrations. This may be the facts of that P123 with most long PPO chains tightly self-assembles into a thermodynamically stable micelle $\left(D_{\text {eff. }}=50 \mathrm{~nm}\right.$, data not shown) and L92 with very short PEO is incorporated into P123 micelle core led to increase the particle size to $100 \mathrm{~nm}$. Similarly, F127 with same PPO of P123 formed $c a$. $200 \mathrm{~nm}$ particles in aqueous solution. The micelle mixed with L92 increased the core size of micelle by adding the PPO of L92, resulting in $c a .300 \mathrm{~nm}$.

\section{Turbidity of Binary Systems}

The turbidity of L92 dispersion and binary systems including L92 (0.1\%) presents in Figure 3. The dispersion of the L92 below $0.1 \%$ was transparent and the turbidity didn't change. The turbidity of $0.5 \%$ L92 dispersion was drastically increased and slightly decreased after 24 hours due to precipitation (data not shown). The binary systems of L92 (0.1\%) including F68, F88, and F98 (0.01 0.1 wt \%) increased the turbidity. This may be due to non-cooperative binding and those secondary aggregations. However, the binary dispersions of L92 (0.1\%) with P85, F127, P123 and P105 (0.01 0.1\%) decreased the turbidity as increase of secondary Pluronics' concentration. Particularly, L92/P123 showed less turbidity and high stability compared to other binary systems. 
(a)

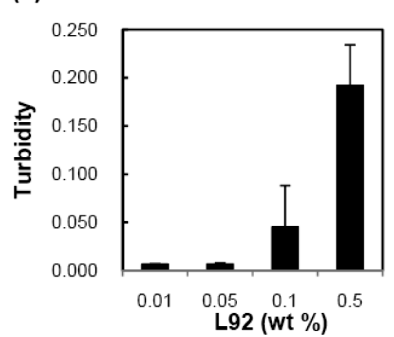

(e)

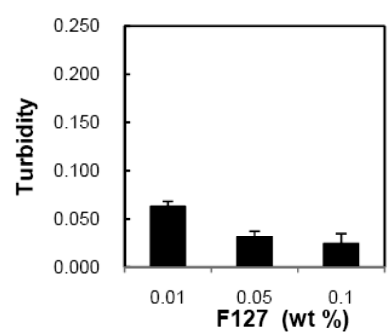

(b)

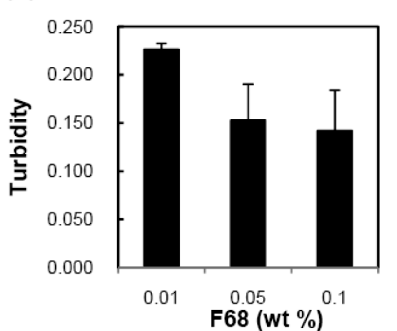

(f)

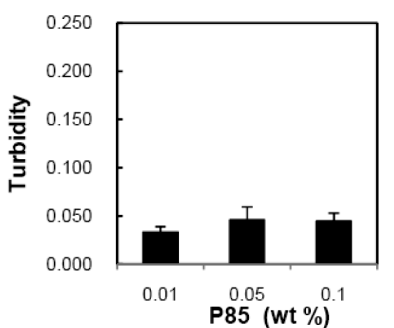

(c)

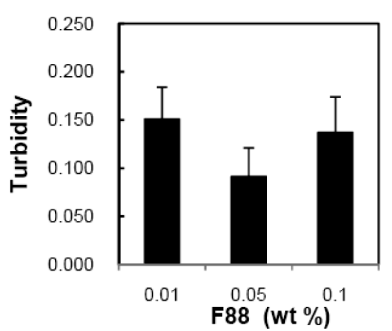

(g)

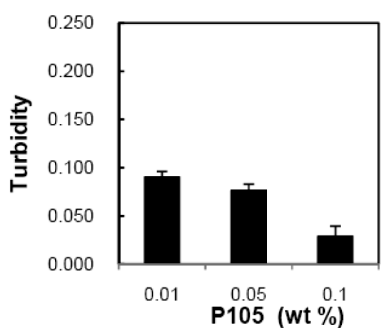

(d)

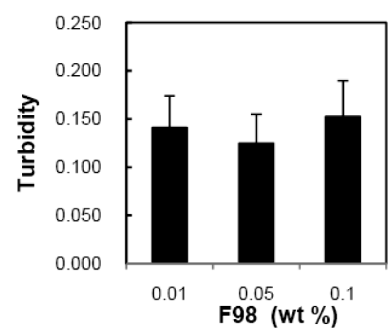

(h)

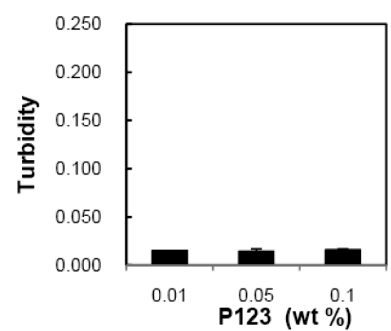

Figure 3. Turbidity of dispersion solutions of (a) mono system L92; and (b) (h) binary systems of 0.1 wt\% L92 admixed with (b) F68 (c) F88 (d) F98 (e) F127 (f) P85 (g) P105 and (h) P123as a function of the concentration. Each point represents average \pm SD (n=3).

(a)

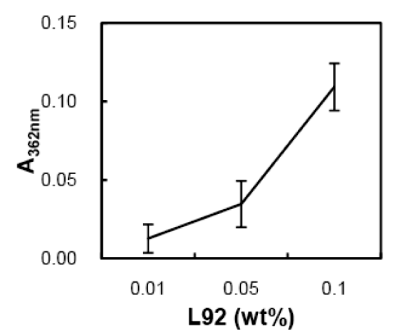

(e)

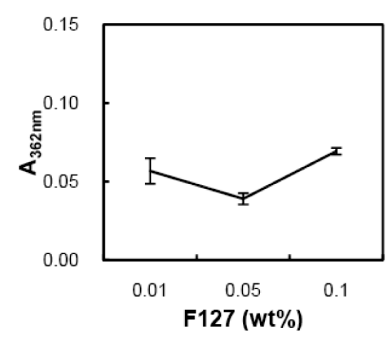

(b)

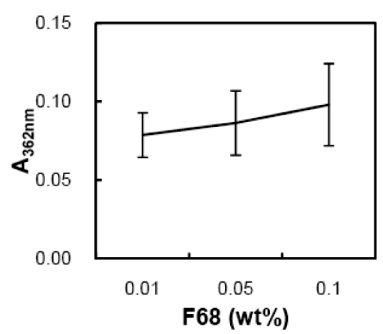

(f)

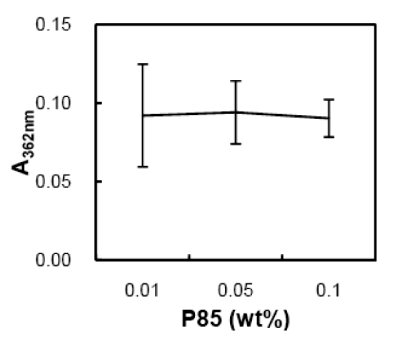

(c)

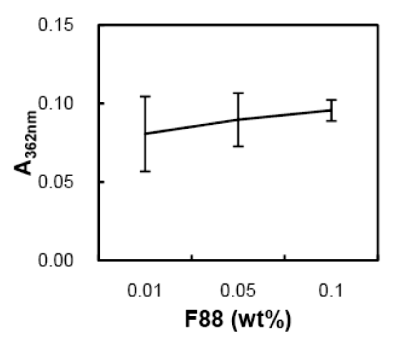

(g)

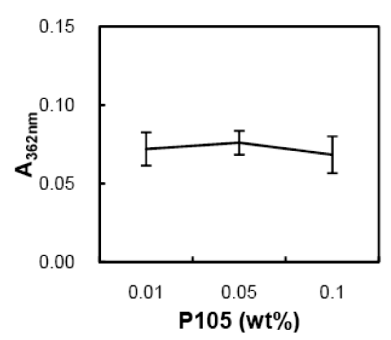

(d)

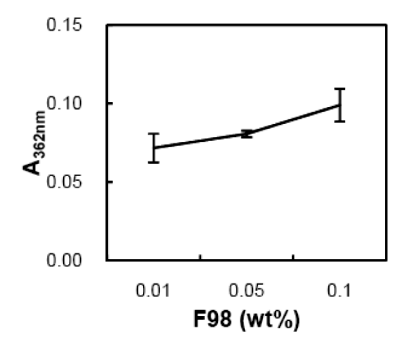

(h)

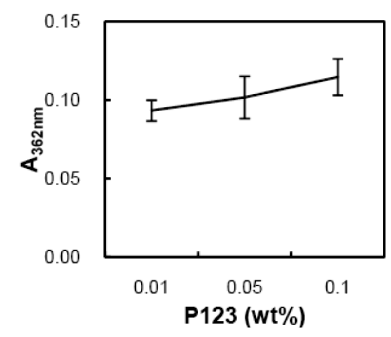

Figure 4. Solubilization of Sudan III in (a) mono system L92; (b) (h) binary systems of 0.1 wt\% L92 admixed with (b) F68 (c) F88 (d) F98 (e) F127 (f) P85 (g) P105, and (h) P123as a function of the concentration. Each point represents average \pm SD (n=3).

\section{Solubilization of Binary Pluronic}

The solubilization capacity of the Pluronic dispersion was evaluated using a water-insoluble dye, Sudan III (Log $\mathrm{P}=5.737$ ), that has a maximum absorbance at $362 \mathrm{~nm}$. The UV/Vis absorbance $(\lambda=362 \mathrm{~nm})$ of Sudan III in Pluronic dispersions versus L92 concentration is shown in Figure 4 (a). The solubilization of Sudan III in L92 was increased with raising L92 concentrations but the particle sizes of L92 was also increased by solubilizing Sudan III. The lamella-forming L92 provides a hydrophobic pool to increase in the solubilization capacity compared to the spherical micelle-forming Pluronic such as F68 and F127 (data not shown). The solubilization capacity of binary systems including L92 prepared in this study was slightly decreased or similar (Figure 4 (b) $\sim$ (h)). However, the particle sizes of the systems didn't change after solubilizing the insoluble dye (data not shown). Interestingly, L92/F127 mixtures drastically decreased the solubilization capacity, which suggested that this combination would not be proper to increase the solubilization of insoluble molecules. On the contrast, the $0.1 \%$ L92/0.1\% P123 presented slightly 
increased solubilization of Sudan III. The incorporation of insoluble dye into the L92/P123 aggregates did not change the particle size, and dispersions remained stable for several days (data not shown). Hence, when the secondary polymer was selected, its cooperative binding to primary polymer, composition of blocks, length of PEO and PPO block, and compatibility between drug and systems should be considered to develop a stable nanocarrier.

\section{Conclusion}

Pluronics are used for pharmaceutical applications such as drug delivery systems, excipients for formulation, and nanosized targeting systems. L92 is hydrophobic, huge aggregation to form the lamellar structure, and unstable in aqueous solution. We tried to develop better systems with L92 by mixing the hydrophilic Pluronics with relatively long PEO block. Among the binary systems, the aggregation of L92/P123 showed less than 100nm size and low turbidity. The dispersion of L92/P123 also presented high stability without change of their size and turbidity for a few weeks. The solubilization capacity of most binary systems didn't increase compared to mono system, L92. Only $0.1 \%$ L92/0.1\% P123 slightly increased the solubilization capacity. This system can be a possible candidate for anticancer nano-sized carriers to reverse the MDR cancer. These nano-sized systems could be useful for anticancer drug delivery systems with simple preparation methods, high stability, and high loading capacity.

\section{Acknowledgements}

This work was supported by the Korea Research Foundation Grant funded by the Korean Government (KRF-2008-331E00463), a grant of the Korean Health Technology R\&D Project, Ministry for Health, Welfare \& Family Affairs, Republic of Korea. (A092018), and a grant from KRIBB Research Initiative Program.

\section{References}

Alakhov, V., Klinski, E., Lemieux, P., Pietrzynski, G., Kabanov, A., 2001. Block copolymeric biotransport carriers as versatile vehicles for drug delivery. Expert Opinion on Biological Therapy. 1, 583-602.

Alakhov, V., Klinski, E., Li, S., Pietrzynski, G., Venne, A., Batrakova, E., Bronitch, T., Kabanov, A.V., 1999. Block copolymerbased formulation of doxorubicin. From cell screen to clinical trials. Colloids Surf., B: Biointerfaces. 16, 113-134.

Allen, C., Maysinger, D., Eisenberg, A., 1999. Nano-engineering block copolymer aggregates for drug delivery. Colloids and Surfaces B: Biointerfaces. 16, 3-27.

Allison, A.C., Byars, N.E., 1986. An adjuvant formulation that selectively elicits the formation of antibodies of protective isotypes and of cell-mediated immunity. J. Immunol. Methods. 95, 157-168.

Allison, A.C., Byars, N.E., 1990. Adjuvant formulations and their mode of action. Semin. Immunol. 2, 369-374.

BASF Pluronic and Tetronic Surfactants. Wyandotte, BASF Corp.

Batrakova, E., Lee, S., Li, S., Venne, A., Alakhov, V., Kabanov, A., 1999a. Fundamental relationships between the composition of pluronic block copolymers and their hypersensitization effect in MDR cancer cells. Pharm Res. 16, 1373-9.

Batrakova, E.V., Lee, S., Li, S., Venne, A., Alakhov, V., Kabanov, A., 1999b. Fundamental relationships between the composition of pluronic block copolymers and their hypersensitization effect in MDR cancer cells. Pharm. Res. 16, 1373-1379.

Batrakova, E.V., Miller, D.W., Li, S., Alakhov, V.Y., Kabanov, A.V., Elmquist, W.F., 2001. Pluronic P85 enhances the delivery of digoxin to the brain: in vitro and in vivo studies. J. Pharmacol. Exp. Ther. 296, 551-557.

Chaibundit, C., Ricardo, N.M.P.S., Costa, F.d.M.L.L., Yeates, S.G., Booth, C., 2007. Micellization and Gelation of Mixed Copolymers P123 and F127 in Aqueous Solution. Langmuir. 23, 9229-9236.

Gaisford, S., Beezer, A.E., Mitchell, J.C., 1997. Diode-Array UV Spectrometric Evidence for Cooperative Interactions in Binary Mixtures of Pluronics F77, F87, and F127. Langmuir. 13, 2606-2607.

Kabanov, A.V., Alakhov, V.Y., 2002. Pluronic block copolymers in drug delivery: from micellar nanocontainers to biological response modifiers. Crit. Rev. Ther. Drug Carrier Syst. 19, 172 .

Kabanov, A.V., Batrakova, E.V., Alakhov, V.Y., 2002. Pluronic block copolymers for overcoming drug resistance in cancer. Adv Drug Deliv Rev. 54, 759-79.

Kataoka, K., Kabanov, A., Eds. (1999). Special Issue: Polymeric Micelles in Biology and Pharmaceutics, Colloids and Surfaces, B: Biointerfaces. Amsterdam, Lausanne, New York, Oxford, Shannon, Tokyo, Elsevier.

Kozlov, M.Y., Melik-Nubarov, N.S., Batrakova, E.V., Kabanov, A.V., 2000. Relationship between pluronic block copolymer structure, critical micellization concentration and partitioning coefficients of low molecular mass solutes. Macromolecules. 33, 3305-3313.

Kwon, G.S., Kataoka, K., 1995. Block copolymer micelles as longcirculating drug vehicles. Advanced Drug Delivery Reviews. 16, 295-309.

Langer, R., 1998. Drug delivery and targeting. Nature. 392, 5-10.

Lee, E.S., Na, K., Bae, Y.H., 2005. Super pH-sensitive multifunctional polymeric micelle. Nano Lett. 5, 325-9.

Lee, E.S., Oh, Y.T., Youn, Y.S., Nam, M., Park, B., Yun, J., Kim, J.H., Song, H.-T., Oh, K.T., 2010. Binary mixing of micelles using Pluronics for a nanosized drug delivery system Colloids 
and Surfaces B: Biointerfaces. in submission.

Lee, E.S., Shin, H.J., Na, K., Bae, Y.H., 2003. Poly(L-histidine)PEG block copolymer micelles and $\mathrm{pH}$-induced destabilization. J Control Release. 90, 363-74.

Nagarajan, R., 1999. Solubilization of hydrocarbons and resulting aggregate shape transitions in aqueous solutions of Pluronic?(PEO-PPO-PEO) block copolymers. Colloids and Surfaces B: Biointerfaces. 16, 55-72.

Oh, K.T., Bronich, T.K., Kabanov, A.V., 2004. Micellar formulations for drug delivery based on mixtures of hydrophobic and hydrophilic Pluronic block copolymers. J Control Release. 94, 411-22.
Oh, K.T., Lee, E.S., Kim, D., Bae, Y.H., 2008. L-histidine-based $\mathrm{pH}$-sensitive anticancer drug carrier micelle: reconstitution and brief evaluation of its systemic toxicity. Int J Pharm. 358, 17783.

Torchilin, V.P., 1999. Polymeric micelles in diagnostic imaging. Colloids and Surfaces B: Biointerfaces. 16, 305-319.

Wang, Y., Yu, L., Han, L., Sha, X., Fang, X., 2007. Difunctional Pluronic copolymer micelles for paclitaxel delivery: Synergistic effect of folate-mediated targeting and Pluronic-mediated overcoming multidrug resistance in tumor cell lines. International Journal of Pharmaceutics. 337, 63-73. 